\title{
Influences of nanoparticles on aquatic organisms: Current situation of nanoparticles effects in aquatic ecosystems
}

\author{
Didem Gökçe 1* $^{*}$ \\ ${ }^{1}$ Science and Arts Faculty, Department of Biology İnönü University, Turkey
}



Keywords: Nanoparticles; Trophic level; Daphnia; Aquatic organisms

\section{Introduction}

In the Anthropocene era, many products used in daily life, industry, medical products, personal care products and cosmetics contain nanoparticles or are made of nanomaterials. Natural, incidental or industrially produced unbound or agglomerated particle-containing materials with a ratio of about $>50 \%$ in the $1-100 \mathrm{~nm}$ size range are defined by the European Commission (2011/696/EU), as nanomaterials [1]. Today, nanotechnology is a rapidly developing interdisciplinary science that can be combined with engineering, biology, chemistry, physics and medicine [2-4]. The physico-chemical characteristics of the nanoparticles are quite different from in larger sizes and agglomerative form. The properties of nanoscale materials have made nanotechnology one of the most important technologies of the twenty-first century. Nanotechnology is now accepted to reaching a market value of 3 trillion dollars by 2020. Nowadays, more than 1800 nano-enabled products are available in the consumer market [3]. Nanoparticles (NPs) are produced and used in large quantities, and are freely released directly / indirectly into different water ecosystems during production, use, discharge, disposal, recycling. On the other words, NPs can enter aquatic ecosystems in different stages: throughout the time of the generation of raw materials and consumer products; throughout the time of the utilize of products containing $\mathrm{NP}$ and subsequently discharge [4,5]. Hence, NPs can impact freshwater and oceans, harms organisms in different aquatic food web levels. Nowadays, the damages of NPs are determined at the food web level. Algae, Daphnia, Artemia (zooplankton), Bivalvia (benthos) and fish represent over the aquatic organism in

This work is licensed under a Creative Commons Attribution License (https://creativecommons.org/licenses/by/4.0/ ) that allows others to share and adapt the material for any purpose (even commercially), in any medium with an acknowledgement of the work's authorship and initial publication in this journal. 
quite different habitats, which make them convenient organisms and models for assessing the environmental impact of NPs [4,6,7]. NPs have critical for two main reasons; firstly, effects on the biochemical and physiological of organisms and secondly, effects on the ecosystem as freshwater and marine systems. The function and balance of the ecosystem is an ecological integrity that affects all living things and nanomaterial and NPs can harm the ecosystem.

NPs carry potential risks to the ecosystem and human health with its wide applications in commercial and different industrial products. Potential toxicity and behavior of NPs can be affected by various factors such as particle charge, size, chemistry and reactivity, surface area, structure and shape, aggregation state and elemental composition.

\section{The structure of nanoparticles}

Nanoparticles are materials with unique structural properties depend on their size, and these properties make nanoparticles advantageous for industrial and medical use. The physical and chemical properties of the particles in the nanoscale are different from those in larger sizes. This is due to the fact that although the nanoscale particles consist of the same atoms as the macro-sized particles, their surface areas increase $[5,7,8]$. In addition to the size of the nanoparticles, properties such as shape, surface load, presence of other materials are among the factors that affect their behavior [4]. Nanoparticles can be classified in different subgroups: Natural, incidental and engineered nanomaterials. The natural nanomaterials are present in ecosystems such as virus and viral capsids. Incidental nanomaterials are accepted as a by-product of various mechanical or industrial processes and reason environmental pollution such as vehicle exhausts and combustion processes. Nanomaterials that are produced and intentionally engineered to have specifically required characteristics are called engineered nanomaterials [7, 9].

Nanoparticles with different properties are collected in two groups as organic NPs (also, metal NPs with an organic coating) and inorganic NPs (also, metal and metal oxide NPs) [6]. The inorganic NPs are accepted to be good materials in some industrial areas such as food, cosmetics and the medical industry (drug delivery and diagnostic imaging) and the advancement of new electronic instruments [6, 10]. Organic NPs consisting of organic compounds (lipids, polymer, carbohydrate, DNA, RNA, proteins and dendrimers) have larger sizes than inorganic NPs. The size of organic particles has a decisive point in their implementations in many areas such as electronics, medicine and environmental science $[6,10]$.

It is known that NPs are released easily into all kinds of ecosystems. On the other hand, especially, the behavior of NPs differs due to their characteristics in the aquatic ecosystems. The chemical composition of water, salinity, organic salts, total suspended solid matter, water circulation patterns and movements in the lake or marine ecosystem [11]. Agglomeration, aggregation and precipitation of NPs are affected by water quality. Based on the studies, the size, shape and surface chemistry or physicochemical properties of the nanoparticles show the level of aggregation, solubility, structural and chemical composition, the importance of the use of NPs and their toxicity with biological systems [10-14]. The size of nanoparticles is a crucial factor in determining their toxicity. NPs size and shape have a direct impact on metabolism. It easily enters the body during respiratory and nutrition and causes significant accumulation in the digestive system. Thence, it plays an important role in the endocytic cellular uptake and biochemical physiological pathways and response of NPs.

As stated above, the shape of NPs is an important feature in the toxicity of NPs, as they can be taken into the cell more easily. Lekamge et al. [4] and Truong et al. [15] indicated that non-spherical shaped NPs (worm, filamentous, or disk-like) could be used effectively in drug transport.

The surface structures of NPs are crucial properties in defining the behavior of NPs. One reason NPs are reactive is their small size and large surface area. This property of NPs and their coatings cause stronger interactions with biological tissues. Therefore, its transfer occurs easily to higher trophic levels [16,17]. Different coating such as carboxylic asides, polymers, polysaccharides, surfactants, organic and inorganic coating can modify surface properties of NPs. The surface charge of NPs helps the adhesion of NPs on cell 
surfaces and significantly impacts its toxicity factor [18].

\section{The effects of NPs on trophic tevel}

Carrying in trophic levels and toxicity of NPs can relate to two groups: non-soluble and water-soluble NPs $[6,7]$. While the water-soluble NPs can accumulate in the aquatic ecosystem, non-soluble NPs can aggregate and take with food particles in the water column or benthic zone. When NPs discharge into the aquatic ecosystem, they undergo a transformation. Depending on the chemical properties of the water, transformations involving different physical, chemical and biological pathways such as agglomeration, absorption and dissolution occur. They cause toxic effects on organisms according to their NPs properties. The exposure time (short term or long term) of the organisms is as important as the NPs concentration [2,10-12,14].

The impacts of NPs on organisms in freshwater ecosystems, especially in standing water habitats such as lake and pool in the river needs to be examined, because of water circulation can observe seasonally due to the absence of water flow. NPs that can agglomerate with organic and inorganic materials accumulate towards the bottom. Even if the lake is in the circulation period, NPs cannot be transported from the habitat as in rivers. The small particle size and water-soluble NPs remain suspended in the water body. As a result, bioaccumulation occurs through the food web (Figure 1).

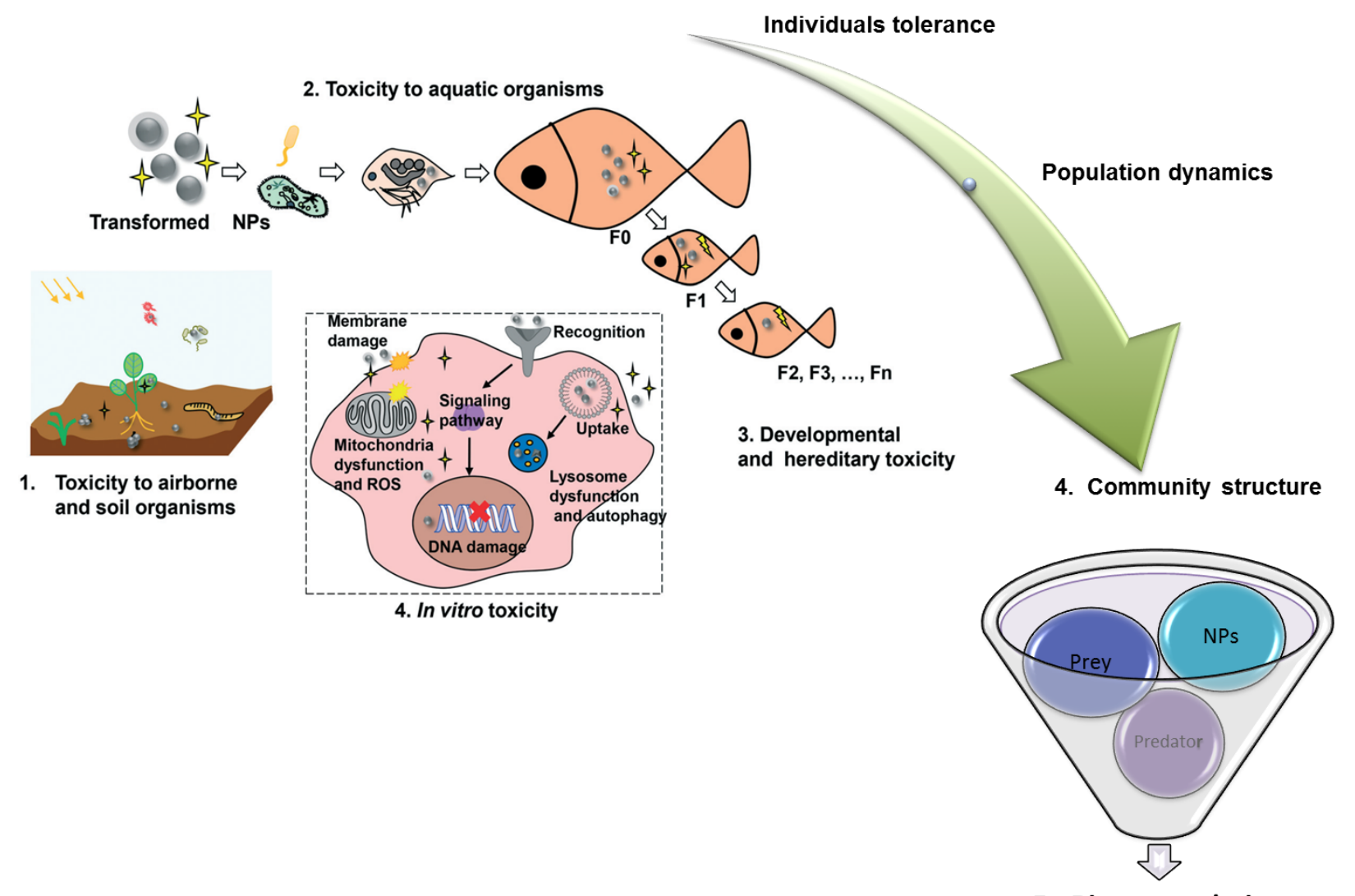

5. Bioaccumulation

Figure 1. The adverse effects of NPs on aquatic community structure and bioaccumulation are summarized. Adapted from $[3,12]$.

Algae, Crustacea, Bivalvia, the rainbow trout (Oncorhynchus mykiss), the zebrafish (Danio rerio) are common models for studying the toxicity of NPs. The transformation of NPs with different characteristic in the freshwater habitats influences toxicity on algae. For instance, magnetic nanoscale zerovalent iron (NZVI) was observed to inhibit the growth of Chlorella vulgaris [19]. For the first time, Zhang et al reported the effect of surface charge of Ag NPs on their accumulation dynamics in Chlorella vulgaris. Polyethylene-coated Ag NPs (PEI-AgNPs) and citrate-coated Ag NPs (Cit-AgNPs) were selected as positively and negatively charged Ag 
NPs (P-AgNPs and N-AgNPs, respectively). Their uptake and elimination dynamics were exanimated at $\mathrm{EC}_{50}$ and $\mathrm{EC}_{10}$ inhibition of growth rate values [20]. $\mathrm{TiO}_{2} \mathrm{NPs}$ is one of the metal oxide nanoparticles, which have been used in personal care products (cosmetics, sunscreen and toothpaste, etc). Mostly anatase and rutile shapes of these NPs which are highly photoreactive forms are used. The photocatalytic potential and toxicity of NPs on Chlorella sp. were studied upon UVA pre-irradiation and UVB pre-irradiation by Roy et al [21]. The authors showed that UVB pre-irradiation of $\mathrm{P} 25 \mathrm{TiO}_{2} \mathrm{NPs}$ caused more toxicity on freshwater algae because of the effect on oxidative stress (SOD, CAT, and APX activities). TiO and $\mathrm{ZnO}$ are widely used metal oxide NPs. Therefore, researches for these two NPs toxicological effects have focused on the model organism Daphnia. In long and short term assays, toxicological impacts of $\mathrm{ZnO} \mathrm{NPs}$ and $\mathrm{TiO}_{2} \mathrm{NPs}$ were studied in detail on reproduction dynamics and population structure of D. magna [11]. In the short time (96h) assays, $\mathrm{LC}_{50}$ values were determined $1.8 \mathrm{mg} \mathrm{L}^{-1} \mathrm{TiO}_{2} \mathrm{NPs}, 0.7 \mathrm{mg} \mathrm{L}^{-1} \mathrm{ZnO} \mathrm{NPs}$, and $0.1 \mathrm{mg} \mathrm{L}^{-1}$ cocktail treatment. In the long time (21d) assays, $\mathrm{LC}_{50}$ value was calculated $1.0 \mathrm{mg} \mathrm{L}^{-1} \mathrm{TiO}_{2} \mathrm{NPs}$, immobilisation and death were recorded. The body morphology and population dynamics were affected negatively depend on increasing concentrations [11].

Banerjee and Roychoudhury [14] stated that a different situation seen in the bio-transfer and bio-accumulation of nanoparticles in the food chain have occurred in biofilm plates. Grazers and detritivores organisms such as snails are fed with biofilm contaminated with $\mathrm{TiO}_{2} \mathrm{NP}_{\mathrm{s}}$ and transfer NPs to the upper trophic levels. In some cases, NPs are generally non-toxic to some microorganisms as they are trapped in the extrapolymeric structure of biofilms. However, leaf-dwelling epiphytic microorganisms are typically sensitive to CuO NPs and Ag NPs [14,22].

Monikh et al. studied that combinations of different physical characteristics as size, shape and the natural organic matter (NOM)-ecocorona of Au NPs impact the attachment of the particles to algae [23]. Spherical $(10,60$ and $100 \mathrm{~nm})$, urchin-shaped $(60 \mathrm{~nm})$, rod-shaped $(10 \times 45,40 \times 60$ and $50 \times 100 \mathrm{~nm})$, and wire-shaped $(75 \times 500,75 \times 3000$ and $75 \times 6000 \mathrm{~nm})$ citrate-coated and NOM-coated Au NPs were applied in the study. The authors demonstrated that the effects of the different morphologic structure of NPs on Pseudokirchinella subcapitata. Among NPs with different sizes and shapes, spherical $10 \mathrm{~nm}$ Au NPs caused membrane detriment to algae. Au NPs with rod-shaped $(10 \times 45 \mathrm{~nm})$ caused cell membrane detriment. Wire-shaped Au NPs caused no membrane detriment to the P. subcapitata [23]. A kind of alter AgNPs makes in the defensive behavior of Daphnia magna maternal and subsequent generation has been studied by Hartman et al [24]. Adult D. magna has the ability to typical anti-predator defense mechanisms when applied to kairomones and AgNPs $(14.9 \pm 2.4 \mathrm{~nm}$ and concentration range, $2.5 \mu \mathrm{g} / \mathrm{L}-20 \mu \mathrm{g} / \mathrm{L})$. On the other hand, their offspring could not show such defensive characteristics. The lack of this adaptive defense mechanism will have a negative effect on D. magna population dynamics and hence potentially on the whole food web in the freshwater ecosystem.

Gökçe et al. demonstrated that the effects of MNPs with different particle sizes on Daphnia population were tested by short term bioassay [2]. According to mortality and morphological measurements, $\mathrm{CuFe}_{2} \mathrm{O}_{4} \mathrm{MNPs}$ were found to be more toxic than the other two MNPs. The concentrations of $\mathrm{CuFe}_{2} \mathrm{O}_{4}, \mathrm{Co} \mathrm{Fe}_{2} \mathrm{O}_{4}$, and Ni $\mathrm{Fe}_{2} \mathrm{O}_{4}$ MNPs drastically affected life span and morphologic growth of D. magna as a result of a short time (96 h) exposure.

Although biosynthesized nanoparticles are identified as green products, the surveys on their toxicity to aquatic food chains are insufficient [18]. Biosynthesized Ag NPs were produced by the reaction of Ag ions with leaf extract of plant Alcea rosea (AR-Ag NPs). The toxic effects of AR-Ag NPs and their pioneers such as Ag ions and coating agent (A. rosea leaf extract) on Chlorella vulgaris, Daphnia magna and Danio rerio, organisms of different trophic levels of a freshwater food web were examined. D. magna was found the most sensitive organism to AR-Ag NPs exposure according to results [18].

The bioaccumulation and biomagnification of nanoparticles can change the reproductive potential, swimming behavior and growth rate in some of the aquatic organisms such as Daphnia and zebrafish (Danio rerio). NPs accumulation on the algal surface could obstruct the uptake of nutrients by algae. Also, nanomaterials can 
play a role as mimic for nutrients by their strong adsorption. While exposure to NPs causes a reduction in body morphology (such as length and width) as the concentration increased, and alter defense behavior in Crustacean population, cause variations of survival, hatching periods and larval morphology, malformations in the fish larvae.

The morphology, physicochemical characteristics and concentration of nanoparticles determine risks on aquatic organisms. Continuity of biochemical pathways and cell structure depend on the kinds of nanoparticles. The previous studies in the literature, the population structure including individual variations shown negatively affect by these NPs amounts. When the community structure of organisms in different trophic steps is adversely affected, it is seen that the aquatic ecosystem balance is disturbed. Monitoring the behavior of these pollutants entering the aquatic ecosystem from point / non-point sources with the population structure of biomonitor organisms plays a key role.

\section{Conclusion}

Nanoparticle materials are used more and more every day due to their industrial advantages. There are potential risks with the discharge of nanoparticles into the freshwater ecosystems. NPs can alter the population dynamics and community structure at the species level that are more sensitive or tolerant to environmental pollution. Moreover, there is limited information regarding nanoparticles' impact on the biota and ecosystem balance/ dynamics.By consuming organisms contaminated with NPs, NPs in the food web are transferred to higher-level organisms. Nanoparticles aggregated in the respiration and digestive system (gill and gut) in considerable concentrations have possible toxic effects. Biomonitor organisms are very sensitive to changes in water quality, hence, reflect ecosystem contamination degrees. It is recommended that the aquatic ecosystem should be monitored long term with the determined organism. Environmental risk assessments approach with three factors that define the resource of the problem: selecting population level endpoints, identifying the ecosystem, and defining the threat or pollution. In this context, more specified and comprehensive environmental risk assessment methods should be implemented for each level of the aquatic ecosystem before wastewater discharge and applying NPs for lake restoration. Consequently, alteration in population dynamics and community structures upon exposure to NPs are crucial in terms of possible indication changes in the ecosystem. NPs use should be limited based on human health and ecosystem balance; and waste water treatment should be performed according to standards.

\section{References}

[1] The European Commission. Commission Recommendations on the definition of nanomaterial (2011/696/EU). 2011:3p.

[2] D. Gökçe, S. Köytepe, I. Özcan, "Assessing short-term effects of magnetite ferrite nanoparticles on Daphnia magna;" Environmental Science and Pollution Research, vol. 27, no. 3, pp. 3189-31504, 2020.

[3] J. Zhang , W. Guo, Q. Li, Z. Wang Z, S. Liu, "The effects and the potential mechanism of environmental transformation of metal nanoparticles on their toxicity in organisms," Environmental Science: Nano, vol. 5, no. 11, pp. 2482-2499, 2018.

[4] S. Lekamge, A. S. Ball, R. Shukla, D. Nugegoda, "The toxicity ofnanoparticles to organisms in freshwater," in: Reviews of environmental contamination and toxicology, Vol. 248, P. de Voogt, Ed. Cham: Springer, 2018, pp. 1-80.

[5] J. D. Almeida, C. E. D. Cardoso, E. Pereira, R. Freitas, "Toxic effects of metal nanoparticles in marine invertebrates", in: Nanostructured materials for treating aquatic pollution engineering materials, G. Gonçalves, P. Marques, Ed. Cham: Springer, 2019, pp. 175-224. 
[6] A. M. Maharramov, U. A. Hasanova, I. A. Suleymanova, G. E. Osmanova, N. E. Hajiyeva, "The engineered nanoparticles in food chain: potential toxicity and effects," SN Applied Sciences, 1362, 2019.

[7] F. Chen, Z. Xiao, L. Yue, J. Wang, Y. Feng, X. Zhu X, Z. Wang, B. Xing, "Algae response to engineered nanoparticles: current understanding, mechanisms and implications," Environmental Science: Nano, pp. 1-14, 2019.

[8] R. Altenburger, W. Brack, R. M. Burgess, W. Busch, B. I. Escher, A. Focks, L. M. Hewitt, B. N. Jacobsen, M. López de Alda, S. Ait-Aissa, T. Backhaus, A. Ginebreda, K. Hilscherová, J. Hollender, H. Hollert, P. A. Neale, T. Schulze, E. L. Schymanski, I. Teodorovic, A. J. Tindall, U. G. de Aragão, B. Vrana, B. Zonja, M. Krauss, "Future water quality monitoring: improving the balance between exposure and toxicity assessments of real-world pollutant mixtures", Environmental Sciences Europe, vol. 31, 12, 2019.

[9] M. Zhang, J. Yang, Z. Cai, Y. Feng, Y. Wang, D. Zhang, X. Pan, "Detection of engineered nanoparticles in aquatic environment: state-of-art and challenges in enrichment, separation and analysis," Environmental Science: Nano, pp. 1-79, 2019.

[10] M. E. Vance, T. Kuiken, E. P. Vejerano, S. P. McGinnis, M. F. Jr. Hochella, D. Rejeski, M. S. Hull, "Nanotechnology in the real world: Redeveloping the nanomaterial consumer products inventory", Beilstein Journal of Nanotechnology, vol. 6, no. 1, pp. 1769-1780, 2015.

[11] D. Gökçe, S. Köytepe, I. Özcan, “Effects of nanoparticles on Daphnia magna population dynamics," Chemistry and Ecology, vol. 34, no. 4, pp. 301-323, 2018.

[12] G. S. Gupta, R. Shanker, A. Dhawan, A. Kumar, "Impact of Nanomaterials on the Aquatic Food Chain", Nanoscience in Food and Agriculture, vol. 5, pp.309-333, 2017.

[13] N. B. Bakaraki, H. S. Erkan, O. G. Engin, M. S. Bilgili, "Nanoparticles in the aquatic environment: usage, properties, transformation and toxicity-A review," Process Safety and Environmental Protection, vol. 130, pp. 238-249, 2019.

[14] A. Banerjee, A. Roychoudhury, "Nanoparticle-Induced Ecotoxicological Risks in Aquatic Environments," Nanomaterials in Plants, Algae and Microorganisms: Concepts and controversies, D. K. Tripathi, P. Ahmad, S. Sharma, D. K. Chauhan, N.K. Dubey, Ed. Elsevier, pp. 129-141, 2019.

[15] N. P. Truong, M. R. Whittaker, C. W. Mak, T. P. Davis, "The importance of nanoparticle shape in cancer drug delivery," Expert Opinion on Drug Delivery, vol.12, pp. 129-142, 2015.

[16] M. Zhang, J. Yang, Z. Cai, Y. Feng, Y. Wang, D. Zhang, X. Pan, "Detection of engineered nanoparticles in aquatic environment: state-of-art and challenges in enrichment, separation and analysis," Environmental Science: Nano, pp. 1-79, 2019.

[17] S. X. T. Liang, L. S. Wong, A. C. T. A. Dhanapal, S. Djearamane, "Toxicity of Metals and Metallic Nanoparticles on Nutritional Properties of Microalgae", Water, Air, \& Soil Pollution, vol. 231, no. 2, pp. 231-252, 2020.

[18] M. Khoshnamvand, Z. Hao, O. O. Fadare, P. Hanachi, Y. Chen, J. Liu, "Toxicity of biosynthesized silver nanoparticles to aquatic organisms of different trophic levels," Chemosphere, vol. 258, 127346, 2020 .

[19] C. Lei, L. Q. Zhang, K. Yang, L. Z. Zhu, D. H. Lin, „Toxicity of iron-based nanoparticles to green algae: Effects of particle size, crystal phase, oxidation state and environmental aging," Environ Pollution, vol. 218, pp. 505-512, 2016.

[20] J. Zhang, Q. Xiang, L. Shen, J. Ling, C. Zhou, J. Hu, L. Chen, "Surface charge-dependent bioaccumulation dynamics of silver nanoparticles in freshwater algae," Chemosphere, vol. 247, 125936, 2020. 
[21] B. Roy, P. K. Suresh, N. Chandrasekaran, A. Mukherjee, "UVB pre-irradiation of titanium dioxide nanoparticles is more detrimental to freshwater algae than UVA pre-irradiation" Journal of Environmental Chemical Engineering, vol. 5, no. 5, 104076, 2020.

[22] M. Bundschuh, F. Seitz, R. R. Rosenfeldt, R. Schulz, "Effects of nanoparticles in fresh waters: risks, mechanisms and interaction," Freshwater Biology, vol. 61, pp. 2185-2196, 2016.

[23] F. A. Monikh, D. Arenas-Lago, P. Porcal, R. Grillo, P. Zhang, Z. Guo Z, "Do the joint effects of size, shape and ecocorona influence the attachment and physical eco(cyto)toxicity of nanoparticles to algae?" Nanotoxicology, vol. 14, no. 3, pp. 310-325, 2020.

[24] S. Hartmann, A. Beasley, D. Mozhayeva, C. Engelhard, K. Witte, "Defective defense in Daphnia daughters: silver nanoparticles inhibit anti-predator defense in offspring but not in maternal Daphnia magna," Scientific Reports, vol. 10, no. 1, 8021, 2020. 